\title{
Comparison of Cobas 6500 and Iris IQ200 fully-automated urine analyzers to manual urine microscopy
}

\author{
Ebubekir Bakan, Nurinnisa Ozturk*, Nurcan Kilic Baygutalp, Elif Polat, Kadriye Akpinar, Emrullah Dorman, Harun Polat, Nuri Bakan \\ Ataturk University, Faculty of Medicine, Department of Medical Biochemistry, Erzurum, Turkey \\ *Corresponding author: nurinnisa.ozturk@gmail.com
}

\begin{abstract}
Introduction: Urine screening is achieved by either automated or manual microscopic analysis. The aim of the study was to compare Cobas 6500 and Iris IQ200 urine analyzers, and manual urine microscopic analysis.

Materials and methods: A total of 540 urine samples sent to the laboratory for chemical and sediment analysis were analyzed on Cobas 6500 and Iris IQ200 within 1 hour from sampling. One hundred and fifty three samples were found to have pathological sediment results and were subjected to manual microscopic analysis performed by laboratory staff blinded to the study. Spearman's and Gamma statistics were used for correlation analyses, and the McNemar test for the comparison of the two automated analyzers.

Results: The comparison of Cobas u701 to the manual method yielded the following regression equations: $\mathrm{y}=-0.12(95 \% \mathrm{Cl}:-1.09$ to 0.67$)+0.78$ (95\% Cl: 0.65 to 0.95$) x$ for WBC and $y=0.06(95 \%$ Cl: -0.09 to 0.25$)+0.66(95 \%$ Cl: 0.57 to 0.73$) x$ for RBC. The comparison of IQ200 Elite to manual method the following equations: $y=0.03(95 \% \mathrm{Cl}:-1.00$ to 1.00$)+0.88(95 \% \mathrm{Cl}: 0.66$ to 1.00$)$ x for WBC and $y=-0.22(95 \% \mathrm{Cl}:-0.80$ to 0.20$)+$ 0.40 (95\% Cl: 0.32 to 0.50) x for RBC. IQ200 Elite compared to Cobas u701 yielded the following equations: $y=-0.95$ (95\% Cl: -2.13 to 0.11$)+1.25$ (95\% Cl: 1.08 to 1.44) x for WBC and $y=-1.20(95 \%$ Cl: -1.80 to -0.30$)+0.80(95 \%$ Cl: 0.55 to 1.00$) x$ for RBC.
\end{abstract}

Conclusions: The two analyzers showed similar performances and good compatibility to manual microscopy. However, they are still inadequate in the determination of WBC, RBC, and EC in highly-pathological samples. Thus, confirmation by manual microscopic analysis may be useful.

Key words: auto-analysis; microscopy; urinalysis

\section{Introduction}

Chemical and microscopic testing of urine samples, known as urinalysis, is one of the most commonly-performed tests in clinical laboratories in order to illuminate several diseases. Urine is a critical health indicator for urinary tract infections, kidney disease and diabetes, which do not exhibit symptoms in early but treatable stages and can result in severe damage if they remain undiagnosed (1-4). The drive towards fully-automated systems for urinalysis has generally been caused by the need to shorten the turnaround time (TAT) and by the increased number of daily samples $(5,6)$. It has been suggested that the importance of the preanalytical stage for total quality be stressed by detailed illustrative advice for specimen collection and that attention is given to emerging automated technology, since automated urinalysis systems allow urine samples to be used as freshly as possible (7). Despite several disadvantages of automated urinalysis systems, they have been introduced widely in clinical laboratories because it is very difficult to standardize urine analysis. However, there is still a need to examine urine samples using manual microscopy (5,8-11). Recently, the advantages and disadvantages of automated urine microscopic analyses have been well documented with the comparison of manual microscopic analysis (12).

Several manufacturers have developed a new generation of more sophisticated automated urinalysis platforms. Automated microscopic and strip 
analyzers have been combined into fully automated workstations. Of these, the Cobas 6500 (Roche, Mannheim, Germany) and Iris IQ200 (Iris Diagnostics, Chatsworth, USA) were used in our study. The aim was to compare Roche Cobas 6500, IRIS IQ200 and manual microscopy. Such a study was obligatory in order to be able to decide which urinalysis platform to purchase for our laboratory.

\section{Materials and methods}

\section{Materials}

Freshly-collected urine samples from 540 out- and in-patients were used in this study. The samples were submitted to our laboratory (Ataturk University, Hospital laboratory, Erzurum, Turkey) for routine diagnostic purposes between September 2015 and March 2016. The ethical approval was not requested, since an informed consent was not necessary for the study, and we ensured patient data privacy as in the case of other patient test results stored in our laboratory information system.

Each primary urine sample was collected in 120$\mathrm{mL}$ sterile Becton Dickinson (BD, Becton Dickinson, Tamse, Switzerland, Ref: 364941) urine containers and aliquoted in three portions of $8 \mathrm{~mL}$ each in 11-mL BD tubes (Becton Dickinson, Tamse, Switzerland, Ref: 364915) without additives/preservatives (i.e. one tube for manual microscopy and one tube for each of the two urine analysis devices). The total analysis duration of each sample was not more than 1 hour. The samples were analyzed as small batches in different sessions and on different days. In one session, we processed as many samples for which it was possible to execute the manual and automated determinations. All results, both chemical and microscopic, from the two analyzers were recorded. In order to evaluate the analytical performances of the workstation, proper control materials were used for precision (between- and within-run variations) and carryover measurements.

The Cobas 6500 platform combines the Cobas u601 and Cobas u701 analyzers, while the Iris IQ200 combines the IChem Velocity and IQ200 Elite. We compared the urine microscopy units
(Cobas u701 and IQ200 Elite) to manual urine microscopic analysis, using similar evaluation parameters (cells/particles per low-power field (LPF) or high-power field (HPF)). In addition, the urine chemical analysis stations, Cobas u601 and IChem Velocity, were also compared.

The urine chemistry profile is analyzed on both devices by reflectance photometry, and the specific gravity by refractometry. The Iris IQ200 Elite evaluates digital images of un-centrifuged urine obtained from laminar flow of the sample, while the Cobas u701 uses specially designed cuvettes for urine centrifugation and visualizes the sediment with light microscope. The obtained HPF digital images are used for microscopic evaluation. Specific recognition software is used in these workstations in order to evaluate the urinary particles.

\section{Methods}

Cobas u601 chemistry module uses test strips cassettes (Cobas u pack; REF 06334601; Roche Diagnostics, Mannheim, Germany) and $0.8 \mathrm{~mL}$ sample from $1.8 \mathrm{~mL}$ dead volume, to analyze erythrocytes (RBC), haemoglobin, leukocytes (WBC), nitrite, ketones, glucose, protein, urobilinogen, bilirubin, $\mathrm{pH}$ and colour with reflectance photometry (at 4 different wavelengths); and clarity with turbidimetry and specific gravity with refractometry. The new technology used in this platform allows the differentiation of intact and lysed erythrocytes up to 50 $\mathrm{RBC} / \mu \mathrm{L}$. For chemical measurement, the sample is aspirated with a pipette and drops are placed onto each reaction pad separately to avoid cross contamination. Following an incubation period, quantitative (specific gravity and $\mathrm{pH}$ ) and semi-quantitative (the remaining parameters) results are reported. The IChem Velocity, the chemistry module of IRIS IQ200, uses the identical measurement principle as the Cobas u601.

Cobas u701, a fully automated microscopic module produced by Roche, re-suspends the sample, pipettes it into a special disposable cuvette, centrifuges the cuvette (resulting in a very thin layer of particles), and captures 15 images of each centrifuged sample. The images are evaluated by a spe- 
cial image-processing algorithm, retrained and reported. The microscopic evaluation rate of Cobas u701 is about 30 seconds for each sample. However, re-classification functionality allows the operator to re-classify any particle. The operator can define cross-check and validation rules. The microscopic unit includes 11 parameters: RBCs, WBCs, squamous epithelial cells, non-squamous epithelial cells, bacteria, hyaline casts, pathological casts, crystals, yeasts, mucus and sperm. Cobas u701 is a reagent-free design, and only uses disposable cuvettes as consumables.

IQ200 Elite, a fully automated microscopic module of Iris, uses a different microscopic examination technique: laminar flow digital imaging technology. The intelligence identification software classifies and quantifies the cells and particles in uncentrifuged urine. In the system, the sample makes a single, laminar flow of the sample through the objective lens of a charged coupling device video camera. The hundreds of captures of a digital camera are evaluated by intelligence identification software, and each particle is classified on the basis of some characteristics such as shape, contrast and texture of the particle.

The quality assessment processes were based on current guidelines (13). Using urine control material level 2 (Liquicheck Urinalysis Control, Lot: 6580, Biorad Laboratories, CA, USA), the intra- and interassay coefficient of variation (CV) of the RBC and WBC counts for Cobas u701 were determined (intra-assay CV was $22.1 \%$ and $26.2 \%$ for WBC and $\mathrm{RBC}$, respectively; inter-assay $\mathrm{CV}$ was $28.8 \%$ and $31.0 \%$ for WBC and RBC, respectively). Control lev- el 1 was a negative control, and no satisfactory figures could be obtained for determinations of microscopic values. Repetitions were 20 for both intra- and inter-assay measurements. No carry-over was detected for Cobas 6500 using control level 1 and 2 in loading the control materials on the analyzer in the order of level 1 - level 2 - level 1.

Of the total 540 samples, only 153 , which were reported as pathological samples by the two automated workstations, were examined with manual microscopy. All microscopic examinations were carried out by the same technician with the same microscope to minimize inter-observer variability. For this purpose, a urine specimen of about $8 \mathrm{~mL}$ was centrifuged at 1500 RPM $(186 \times \mathrm{g})$ for $5 \mathrm{~min}$ (Allegra x-30R, Beckman Coulter, Brea, USA), and the sediment was obtained for microscopic examination $(6,13)$. The microscopic examinations were executed within 1 hour after sampling. After slide preparation, it was evaluated using a light microscope (Olympus CX21FS1, Olympus, Japan) at magnifications of $\times 100$ (LPF) for the crystals and epithelial cells and $x 400$ (HPF) for RBC and WBC. Together with minor modifications in the evaluation criteria or cut-off values, the particles were counted per field, and the results were classified as shown in Table 1 (14-17). Manual microscopy was taken as the reference for urine sediment evaluations.

\section{Statistical analysis}

The Kolmogorov-Smirnov test was used to verify the normality of the parameters. For correlation analyses, Gamma and Spearman's correlations

TABLE 1. Semi-quantitative reference values for urine sediment analysis

\begin{tabular}{lccccc}
\hline \multirow{2}{*}{\multicolumn{1}{c}{ Parameters }} & \multicolumn{5}{c}{ Ranges } \\
\cline { 2 - 6 } & Negative & \multicolumn{4}{c}{ Positive } \\
\cline { 2 - 6 } & & Few & Moderate & High & Many \\
\hline WBC (cells / HPF) & $0-5$ & $6-10$ & $11-20$ & $21-50$ & $\geq 51$ \\
RBC (cells / HPF) & $0-5$ & $6-10$ & $11-20$ & $21-50$ & $\geq 51$ \\
Epithelial cells (cells / LPF) & Negative & Few & Moderate & High & - \\
Crystals (particle / LPF) & Negative & Positive & - & - & - \\
\hline
\end{tabular}

WBC - white blood cells. RBC - red blood cells. HPF - high power field (x400). LPF - low power field (x100). 
were used to compare nonparametric data. Gamma statistics analysis was applied to calculate the correlation depending on the matching and nonmatching for the pairs of observation in the crosstables. The results of the 3 methods were evaluated and categorized on the basis of clinical decision. For example, for glucose the numerical categorization was made as: negative (0), trace (1), onepositive (2), two-positive (3) and three-positive (4). The McNemar's test was used for the comparison of the two automated analyzers in respect of the results affecting clinical decision. The results of the two analyzers and manual microscopy were compared using Passing-Bablok and Bland-Altman plots for association and differences. Kappa (K) analysis was used to demonstrate the agreement of comparison results of the three microscopic methods. Statistical analysis was made using the SPSS 20.0 program (SPSS, Chicago, USA) and MedCalc Statistical Software (version 12, MedCalc Software, Mariakerke, Belgium). A value of $P<0.05$ was considered statistically significant.

\section{Results}

Table 2 shows the diagnostic sensitivity and specificity of Cobas u701 and IQ200 Elite. In this evaluation, if a sample had positivity in both manual and automated microscopy, the automated result was considered as true positive, and if a sample was determined as positive in manual and as negative in the automated system, it was deemed false negative. On the other hand, if a sample had negativity in both manual and automated microscopy, the automated result was considered as true negative, and if a sample was determined as negative in manual and positive in the automated system, it was considered false positive. The calculated sensitivity and specificity percentages were considered as evaluation criteria. As a result, the Cobas u701 (93\%) and IQ200 Elite (92\%) had similar sensitivity for leukocyte counting, while the Cobas u701 (87\%) was more specific for leukocyte counting than IQ200 Elite (71\%). The Cobas u701 (81\%) was more specific for erythrocyte counting than IQ200 Elite (63\%). However, IQ200 Elite (90\%) was more

TABLE 2. Diagnostic sensitivity and specificity of Cobas 6500 and IQ200 for urine sediment

\begin{tabular}{ccccc}
\hline Parameters & Sensitivity (\%) & Specificity (\%) & PPV (\%) & NPV (\%) \\
\hline WBC (Cobas 6500) & 93 & 87 & 93 & 85 \\
RBC (Cobas 6500) & 82 & 81 & 80 & 74 \\
WBC (IQ200) & 92 & 71 & 83 & 75 \\
RBC (IQ200) & 90 & 63 & 65 & 76 \\
\hline
\end{tabular}

WBC - white blood cells. RBC - red blood cells. PPV - positive predictive value. NPV - negative predictive value. Manual urine sediment analysis was used as reference.

TABLE 3. Correlations between manual and automated microscopy

\begin{tabular}{ccccc}
\hline Cells and particles by manual microscopy \\
(N = 153) & \multicolumn{2}{c}{ Cobas 6500 } & \multicolumn{2}{c}{ IQ200 } \\
\cline { 2 - 5 } WBC (cells / HPF) & $\mathbf{r}$ & $\mathbf{P}$ & $\mathbf{r}$ & $\mathbf{P}$ \\
RBC (cells / HPF) & 0.82 & 0.001 & 0.81 & 0.001 \\
SEC (cells / LPF) & 0.59 & 0.001 & 0.65 & 0.001 \\
NEC (cells / LPF) & 0.73 & 0.001 & 0.70 & 0.001 \\
Crystal (particles / LPF) & 0.43 & 0.001 & 0.092 & 0.001 \\
\hline
\end{tabular}

WBC - white blood cells. RBC - red blood cells. SEC - squamous epithelial cells. NEC - non-squamous epithelial cells. HPF - high power field (x400). LPF - low power field (x100). $r$ - correlation coefficient. $P<0.05$ was considered statistically significant. 
sensitive for erythrocyte counting than the Cobas u701 (82\%).

Correlations ranged from very good to poor between manual microscopy and the two fully automated urine microscopy analyzers especially for RBC, WBC, squamous epithelial cells and crystals. No correlation was present in non-squamous epithelial cells between the manual microscopy and IQ200 Elite (Table 3).

Correlations found between the two automated microscopy units (Cobas u701 and IQ200 Elite) are presented in Table 4. Correlations between Cobas $\mathrm{u} 601$ and IChem Velocity for $\mathrm{pH}(\mathrm{r}=0.77)$ and specific gravity ( $r=0.92)$ were determined, and there was a good concordance in glucose $(0.88)$, protein (0.89), urobilinogen (0.44), ketones (0.58), nitrite (0.81), blood (0.87), and leukocyte (0.92) determinations between Cobas u601 and IChem Velocity chemistry units. Similarly, the microscopic and strip results for WBC and RBC counts were comparable for the two automated devices $(r=0.74$ and 0.65 for WBC and RBC counts for Cobas 6500, respectively; $r=0.74$ and 0.76 for $\mathrm{WBC}$ and $\mathrm{RBC}$ counts of Iris IQ200, respectively). No correlations were present for non-squamous epithelial cells and bilirubin between the two chemistry units (Table 4).

Gamma statistics and the McNemar's test were only applied to microscopic results of WBC for the three methods because the WBC counts were selected as an example and it is of importance in clinical decision making. Table 5 summarizes the numbers of positivity and negativity rates of WBC counts of manual microscopy, Cobas u701, and IQ 200 Elite and the correlations. Gamma statistics was applied to explain the clinically positive vs. negative results. All parameters were categorized on the basis of the positivity and negativity degree, and the numerical values of the different positive results were taken. As a result of data analyses associated with a Gamma statistics value of 0.99 and considering the cut-off value of $<5$ WBC / $\mathrm{HPF}$, there was a significant difference in the comparison of manual microscopy to Cobas u701 (McNemar test; $\mathrm{P}<0.001$ ). For this comparison, all clinical diagnoses may have been affected by nonconcordant results at a rate of $13.3 \%$. The compari-
TABLE 4. Correlations between the Cobas 6500 and Iris IQ200 automated urine analyzers

\begin{tabular}{lcc}
\hline \multicolumn{3}{c}{ Cobas u601 vs IChem Velocity } \\
\hline Strip parameters (N = 540) & r & P \\
\hline Erythrocytes (x 106/L) & 0.87 & 0.001 \\
Leukocytes $(\times 106 / L)$ & 0.92 & 0.001 \\
Nitrite $(\mu \mathrm{mol} / \mathrm{L})$ & 0.81 & 0.001 \\
Ketones $(\mathrm{mmol} / \mathrm{L})$ & 0.58 & 0.001 \\
Glucose $(\mathrm{mmol} / \mathrm{L})$ & 0.88 & 0.001 \\
Protein $(\mathrm{g} / \mathrm{L})$ & 0.89 & 0.001 \\
Urobilinogen $(\mu \mathrm{mol} / \mathrm{L})$ & 0.44 & 0.001 \\
Bilirubin $(\mu \mathrm{mol} / \mathrm{L})$ & 0.19 & 0.020 \\
pH $(\mathrm{pH}$ units) & 0.77 & 0.001 \\
Color & 0.71 & 0.001 \\
Clarity & 0.72 & 0.001 \\
Specific gravity $(\mathrm{kg} / \mathrm{L})$ & 0.92 & 0.001 \\
\hline
\end{tabular}

\begin{tabular}{lcc}
\hline \multicolumn{3}{c}{ Cobas u701 vs IQ200 Elite } \\
\hline Cellular elements (N = 540) & r & P \\
\hline WBC (cells / HPF) & 0.81 & 0.001 \\
RBC (cells / HPF) & 0.61 & 0.001 \\
SEC (cells / LPF) & 0.73 & 0.001 \\
NEC (cells / LPF) & 0.16 & 0.049 \\
Crystal (particles / LPF) & 0.46 & 0.001 \\
\hline COBAS - 6500 & $\mathbf{r}$ & $\mathbf{P}$ \\
(Strip vs microscopy comparison) & 0.74 & 0.001 \\
\hline WBC (x 106/L) & 0.65 & 0.001 \\
RBC (x 106/L) & $\mathbf{r}$ & $\mathbf{P}$ \\
\hline IRIS IQ200 & & \\
(strip vs microscopy comparison) & & 0.001 \\
\hline WBC (x 106/L) & 0.74 & 0.001 \\
RBC (x 106/L) & 0.76 &
\end{tabular}

HPF - high power field (x400). LPF - low power field (x100). WBC - white blood cells. RBC - red blood cells. SEC squamous epithelial cells. NEC - non-squamous epithelial cells. $r$ - correlation coefficient. $P<0.05$ was considered statistically significant.

son of manual microscopy to IQ200 Elite for WBC counts yielded Gamma correlation of 0.99 , and similarly, there was a significant difference in this comparison (McNemar test; $\mathrm{P}<0.001$ ) and a nonconcordancy of $13.3 \%$ clinically. When the microscopy results of two devices, Cobas u701 and IQ200 Elite, were compared, the Gamma statistics was 
TABLE 5. Gamma statistic comparisons of leukocyte counts between manual method and automated urine analyzers

\begin{tabular}{|c|c|c|c|c|c|c|}
\hline \multirow{2}{*}{$\begin{array}{c}\text { WBC by manual microscopy, } \\
\text { cells / HPF }\end{array}$} & \multicolumn{6}{|c|}{ WBC by Cobas u701, cells / HPF } \\
\hline & $0-4$ & $5-10$ & $11-20$ & $21-50$ & $\geq 51$ & Total \\
\hline $0-4$ & 426 & 4 & 2 & 1 & 1 & 434 \\
\hline $5-10$ & 7 & 9 & 10 & 2 & 4 & 32 \\
\hline $11-20$ & 2 & 1 & 4 & 6 & 4 & 17 \\
\hline $21-50$ & 0 & 1 & 1 & 9 & 6 & 17 \\
\hline$>51$ & 1 & 1 & 2 & 1 & 35 & 40 \\
\hline Total & 436 & 16 & 19 & 19 & 50 & 540 \\
\hline \multirow{2}{*}{$\begin{array}{c}\text { WBC by manual microscopy, } \\
\text { cells / HPF }\end{array}$} & \multicolumn{6}{|c|}{ WBC by IQ 200 Elite, cells / HPF } \\
\hline & $0-4$ & $5-10$ & $11-20$ & $21-50$ & $>50$ & Total \\
\hline $0-4$ & 415 & 10 & 6 & 2 & 1 & 434 \\
\hline $5-10$ & 8 & 14 & 7 & 2 & 1 & 32 \\
\hline $11-20$ & 2 & 5 & 4 & 5 & 1 & 17 \\
\hline $21-50$ & 0 & 1 & 2 & 12 & 2 & 17 \\
\hline$>51$ & 1 & 0 & 0 & 4 & 35 & 40 \\
\hline Total & 426 & 30 & 19 & 25 & 40 & 540 \\
\hline \multirow{2}{*}{$\begin{array}{c}\text { WBC by IQ } 200 \text { Elite, cells / } \\
\text { HPF }\end{array}$} & \multicolumn{6}{|c|}{ WBC by Cobas u701, cells / HPF } \\
\hline & $0-4$ & $5-10$ & $11-20$ & $21-50$ & $>50$ & Total \\
\hline $0-4$ & 417 & 6 & 1 & 1 & 1 & 426 \\
\hline $5-10$ & 11 & 8 & 9 & 0 & 2 & 30 \\
\hline $11-20$ & 5 & 2 & 7 & 3 & 2 & 19 \\
\hline $21-50$ & 1 & 0 & 2 & 13 & 9 & 25 \\
\hline$>51$ & 2 & 0 & 0 & 2 & 36 & 40 \\
\hline Total & 436 & 16 & 19 & 19 & 50 & 540 \\
\hline
\end{tabular}

WBC - white blood cells. HPF - high power field (x400)

found to be 0.99 (McNemar test; $P<0.001$ ), and the non-concordancy was $13.6 \%$ on the basis of clinical decision-making.

There was substantial agreement between comparison pairs: manual microscopy and Cobas $\mathrm{u} 701$ ( $K=0.69 ; P<0.001)$, manual microscopy and IQ200 Elite $(\mathrm{K}=0.69 ; \mathrm{P}<0.001)$, and IQ200 Elite and Cobas $\mathrm{u} 701(\mathrm{~K}=0.69 ; \mathrm{P}<0.001)$, using $\mathrm{K}$ analysis.

As seen in Figures 1a, 2a, 3a, 4a, 5a and 6a, very close equivalent slopes were observed applying Passing-Bablok regression fits. Most of the comparisons yielded slopes of around 1.000 and nearzero intercept (Table 6). Each automated microscopy method was compared with the manual meth- od on the basis of the slope and intercept of the Passing-Bablok regression line and these details are shown in Table 6 . All the regressions mentioned above showed good concordance between manual microscopy and IQ200 Elite and Cobas u701.

Bland-Altman plots showing the compatibility between the microscopic evaluations are shown in Figures 1b, 2b, 3b, 4b, 5b and 6b. An acceptable performance of both automated microscopy units in WBC and RBC determinations can easily be seen when one considers the results of the Bland-Altman difference plots and the biases. 

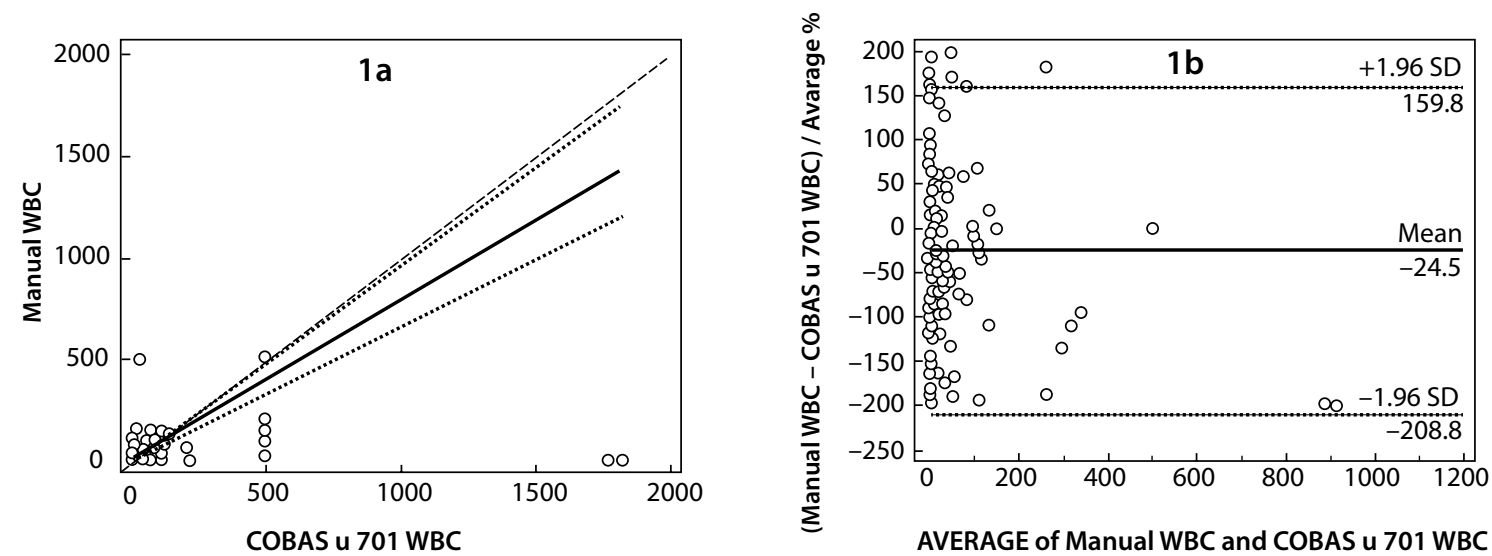

Figure 1. a: Passing-Bablock regression fits for comparisons of manual microscopy and Cobas u701 For WBC count. Solid line - regression line. Dashed lines - $95 \% \mathrm{Cl}$ for the regression line. Dotted line - identity line $(\mathrm{X}=\mathrm{Y}) .95 \% \mathrm{Cl}-95 \%$ confidence interval.

b: Bland-Altman plot shows the comparability of the WBC results obtained with manual microscopy and Cobas u701. Solid line (Mean) - mean difference. Dashed lines (SD) - standard deviation.
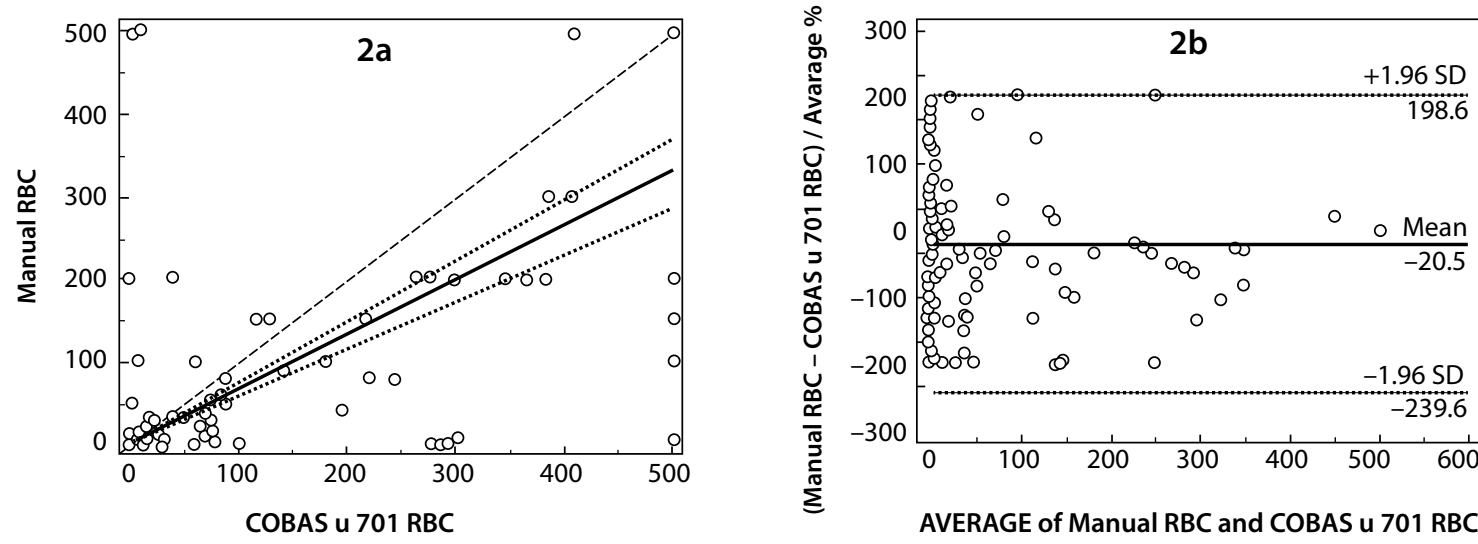

Figure 2. a: Passing-Bablock regression fits for comparisons of manual microscopy and Cobas u701 for RBC count. Solid line - regression line. Dashed lines - 95\% Cl for the regression line. Dotted line - identity line $(X=\mathrm{Y}) .95 \% \mathrm{Cl}-95 \%$ confidence interval. b: Bland-Altman plot shows the comparability of the RBC results obtained with manual microscopy and Cobas u701. Solid line (Mean) - mean difference. Dashed lines (SD) - standard deviation.
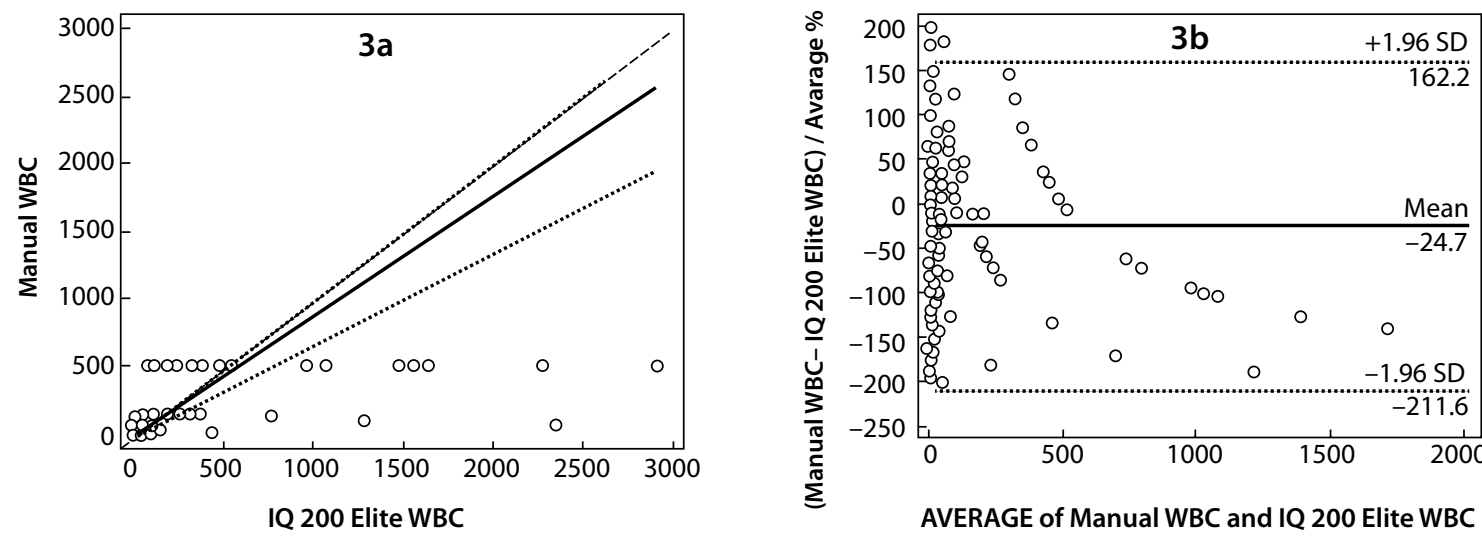

FIGURE 3. a: Passing-Bablock regression fits for comparisons of manual microscopy and IQ200 Elite for WBC count. Solid line - regression line. Dashed lines - $95 \% \mathrm{Cl}$ for the regression line. Dotted line - identity line $(\mathrm{X}=\mathrm{Y}) .95 \% \mathrm{Cl}-95 \%$ confidence interval. b: Bland-Altman plot shows the comparability of the WBC results obtained with manual microscopy and IQ200 Elite. Solid line (Mean) - mean difference. Dashed lines (SD) - standard deviation. 

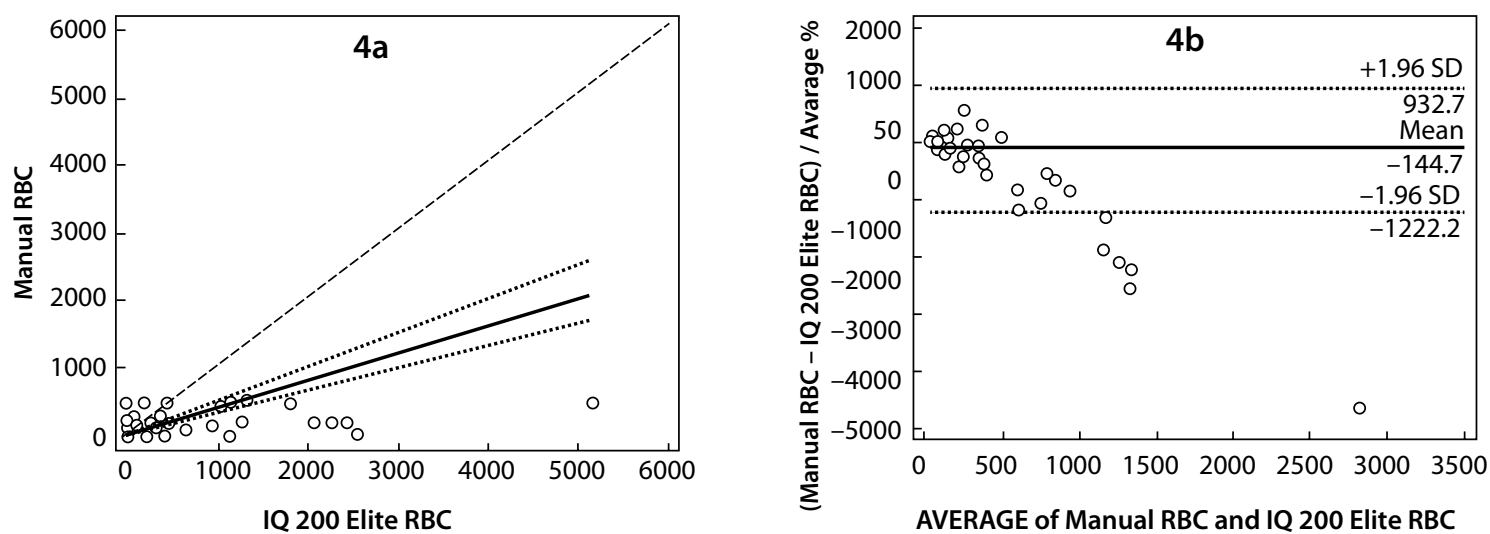

FIGURE 4. a: Passing-Bablock regression fits for comparisons of manual microscopy and IQ200 Elite for RBC count. Solid line - regression line. Dashed lines - $95 \% \mathrm{Cl}$ for the regression line. Dotted line - identity line $(\mathrm{X}=\mathrm{Y}) .95 \% \mathrm{Cl}-95 \%$ confidence interval.

b: Bland-Altman plot shows the comparability of the RBC results obtained with manual microscopy and IQ200 Elite. Solid line (Mean) - mean difference. Dashed lines (SD) - standard deviation.
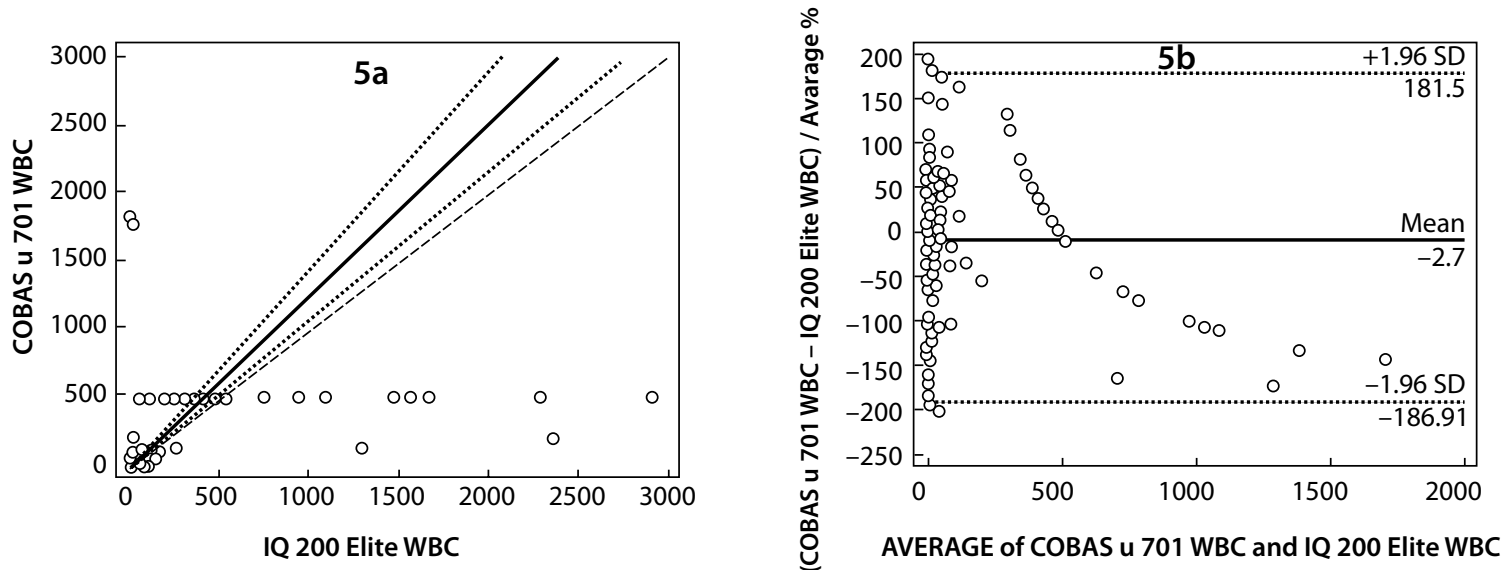

Figure 5. a: Passing-Bablock regression fits for comparisons of manual microscopy and Cobas u701 and IQ200 Elite for WBC count. Solid line - regression line. Dashed lines - $95 \% \mathrm{Cl}$ for the regression line. Dotted line - identity line $(\mathrm{X}=\mathrm{Y}) .95 \% \mathrm{Cl}-95 \% \mathrm{confidence}$ interval.

b: Bland-Altman plot shows the compatibility of the WBC results obtained with Cobas u701 and IQ200 Elite. Solid line (Mean) - mean difference. Dashed lines (SD) - standard deviation.
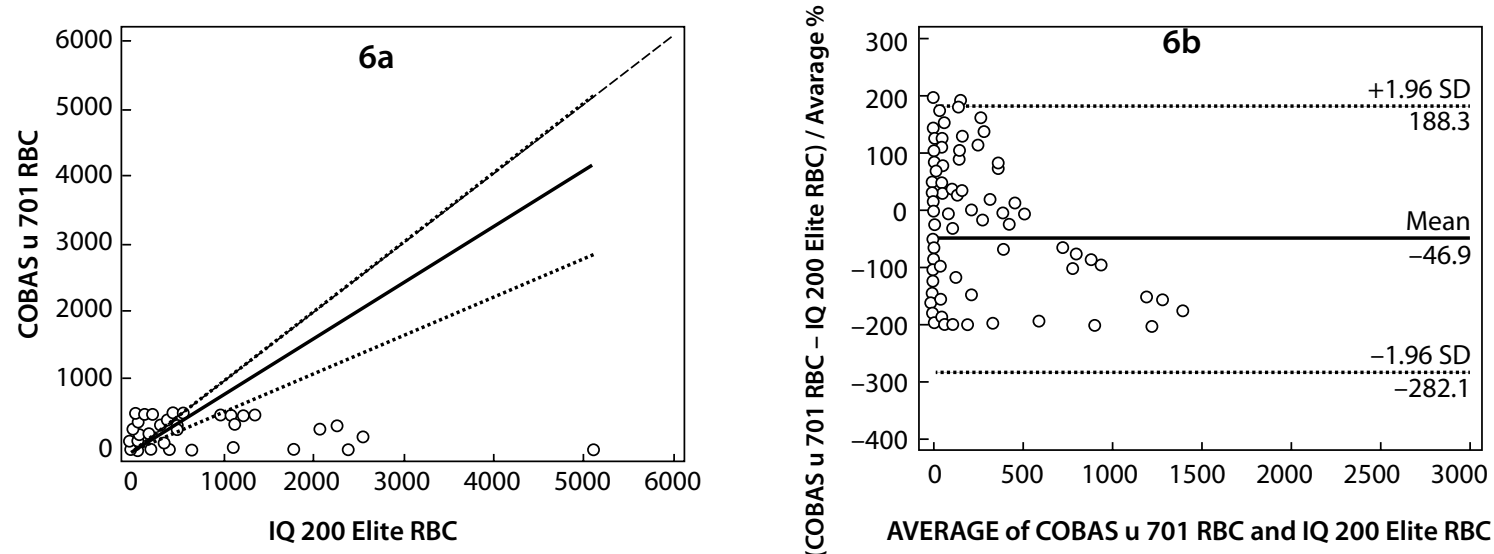

FIGURE 6. a: Passing-Bablock regression fits for comparisons of Cobas u701 and IQ200 Elite for RBC count. Solid line - regression line. Dashed lines - $95 \% \mathrm{Cl}$ for the regression line. Dotted line - identity line $(X=\mathrm{Y}) .95 \% \mathrm{Cl}-95 \%$ confidence interval.

b: Bland-Altman plot shows the compatibility of the RBC results obtained with Cobas u701 and IQ200 Elite. Solid line (Mean) - mean difference. Dashed lines (SD) - standard deviation. 
TABLE 6. Details of the Passing and Bablok regression analysis

\begin{tabular}{|c|c|c|}
\hline Statistical analysis & $\begin{array}{c}\text { Cobas u701 WBC (Variable x) } \\
\text { Manual WBC (Variable y) } \\
\text { (Cells / HPF) }\end{array}$ & $\begin{array}{c}\text { Cobas u701 RBC (Variable x) } \\
\text { Manual RBC (Variable y) } \\
\text { (Cells / HPF) }\end{array}$ \\
\hline Regression equation & $y=-0.12(-1.09$ to 0.67$)+0.78(0.65$ to 0.95$) x$ & $y=0.06(-0.09$ to 0.25$)+0.66(0.57$ to 0.73$) x$ \\
\hline \multirow[t]{2}{*}{ Cusum test for linearity } & $P<0.05$ & $P<0.01$ \\
\hline & $\begin{array}{c}\text { IQ } 200 \text { Elite WBC (Variable x) } \\
\text { Manual WBC (Variable y) } \\
\text { (Cells / HPF) }\end{array}$ & $\begin{array}{c}\text { IQ } 200 \text { Elite RBC (Variable x) } \\
\text { Manual RBC (Variable y) } \\
\text { (Cells / HPF) }\end{array}$ \\
\hline Regression equation & $y=0.03(-1.00$ to 1.00$)+0.88(0.66$ to 1.00$) x$ & $y=-0.22(-0.80$ to 0.20$)+0.40(0.32$ to 0.50$) x$ \\
\hline \multirow[t]{2}{*}{ Cusum test for linearity } & $P>0.10$ & $P>0.05$ \\
\hline & $\begin{array}{c}\text { IQ } 200 \text { Elite WBC (Variable x) } \\
\text { Cobas u } 701 \text { WBC (Variable y) } \\
\text { (Cells / HPF) }\end{array}$ & $\begin{array}{l}\text { IQ } 200 \text { Elite RBC (Variable x) } \\
\text { Cobas u } 701 \text { RBC (Variable y) } \\
\text { (Cells / HPF) }\end{array}$ \\
\hline Regression equation & $y=-0.95(-2.13$ to 0.11$)+1.25(1.08$ to 1.44$) x$ & $y=-1.20(-1.80$ to 0.30$)+0.80(0.55$ to 1.00$) x$ \\
\hline Cusum test for linearity & $P<0.05$ & $P>0.05$ \\
\hline
\end{tabular}

HPF - high power field (x400). WBC - white blood cells. RBC - red blood cells. Regression equations are presented as $y=a(95 \%$ $\mathrm{Cl})+\mathrm{b}(95 \% \mathrm{Cl}) \mathrm{x} .95 \% \mathrm{Cl}$ - confidence intervals of $95 \%$. a - regression line's intercept. $\mathrm{b}$ - regression line's slope. $\mathrm{P}<0.05$ was considered statistically significant.

\section{Discussion}

The microscopy units investigated (i.e. Cobas u 701 and IQ200 Elite) showed very good correlation for WBC, good correlation for RBC and squamous epithelial cells, and poor correlation for crystals. The correlations between Cobas u601 and Iris IChem Velocity were very good for $\mathrm{pH}$ and specific gravity, and there was good concordance in all the parameters tested, except for urobilinogen and ketones. The automated microscopic results obtained using Cobas u701 exhibited very good compatibility for WBC counts with the manual method, while the IQ200 Elite exhibited good compatibility with the manual method for RBC counts. Similarly, the microscopic and strip results for WBC and RBC parameters were comparable in the two automated devices (good for both parameters in both devices). The between- and withinday precisions and carry-over measurements of Cobas 6500 were satisfactory. In clinical decisionmaking, non-concordances lower than $13.6 \%$ in WBC parameter were observed for manual microscopy, Cobas u701, and IQ 200 Elite.

Manual microscopic analysis of urine sediment is very difficult to standardize, since both pre-exami- nation and examination phases are the source of non-standard processes, resulting in false positive and false negative results (18). In order to standardize urinalysis and improve TAT and reliability, the manufacturers of urine analytical systems have developed fully-automated, consolidated urinalysis systems. It has been reported that a proximity to manual microscopic results could be achieved by developing fully-automated urinalysis systems (19-21), and a number of studies have been conducted comparing manual and automated urinalysis (14-16,22-26).

This study can be considered of value as it is the first study to compare Iris IQ200 and Cobas 6500 . Yuksel et al. compared the fully automated urine sediment analyzers H800-FUS100 and LabumatUrised with manual microscopy, and Akin et al. reported that two automated techniques, the UriSed and IQ200, have compatible results with each other and with manual microscopy, and that the confirmation of pathological results of the automated system by manual examination may be needed $(15,16)$. It has also been reported that the automated systems are not completely free of error and 
that one must consider the strip analysis results when interpreting automated microscopic reports, resulting in reduction of test result errors (27).

The negative and the positive predictive values of the two systems could have been determined more sensitively if the KOVA cell chamber system had been used, since semi-quantitative results for manual microscopy were determined in this study, which was a limitation of the present study. Particle count repeatability of several urinalysis systems as the between-day and within-day precisions has been reported previously $(15,16)$. The betweenday and within-day precisions of Cobas 6500 were very similar to the findings of those studies. The carry-over study for Cobas 6500 was made using level 1 and 2 controls, and no carry-over was detected. The compatibility of two automated microscopic units with manual microscopy was similar for all formed elements (RBC, WBC, squamous epithelial cells, and crystals). The strip test results of the two urinalysis platforms were very compatible with each other. Parameters as bilirubin and nonsquamous epithelial cells, showing non-concordance, had low positivity rates. The results of RBC and WBC of strip and microscopy units of both the automated devices were comparable.

Akin et al. (16) applied the Gamma statistics and McNemar test to the microscopic results of WBC and RBC, and they compared manual and automated microscopic determinations in respect of clinically positive vs negative results. They reported non-concordances lower than $10 \%$ in general in clinical decision-making (16). Considering a manual microscopy cut-off value of $<5 \mathrm{WBC} / \mathrm{HPF}$ for Cobas u701 and IQ200 Elite, non-concordances lower than $13.6 \%$ in general in clinical decisionmaking were observed in the current study. The difference between the presented non-concordancies in the present study compared to that of Akin et al. (16) may have been caused by the fact that they used the KOVA method for manual microscopic analyses. Kappa analysis made for manual and Cobas u701, manual and IQ200 Elite, and IQ200 Elite and Cobas u701 comparisons showed substantial agreement between each other.
Although the diagnostic sensitivity and specificity of WBC and RBC counts by Cobas u701 and IQ200 Elite were different from each other, all percentages were within the acceptable range as mentioned above. The Passing-Bablok regression analyses showed good compatibility between manual microscopy and the two automated devices, whereas the Bland-Altman plots demonstrated the compatibility between the microscopic evaluations. An acceptable performance of both the automated microscopy units in WBC and RBC determinations can easily be seen from the Bland-Altman difference plots and the biases.

It is an advantage that the well-trained operators of the fully automated urine devices have the possibility to intervene in the results of these instruments before reporting. The operator is able to examine controversial images and decide which particles should be reported.

The limitation of the present study may be stated as the fact that we were not able to use KOVA cell chamber system as mentioned above. Furthermore, more pathological samples could have been included in the study in order to provide more proper statistical evaluation.

In conclusion, the two urinalysis platforms exhibited similar performances and the microscopic examination results of Cobas $u 701$ had good agreement with those of the manual method. In spite of minor differences in the technologies, these workstations provide standardized processes and improved TAT in urinalysis, which is very important for patient safety and for pre-analytic and analytic error reduction. However, they are still inadequate for satisfactory determination and classification of some sediment particles such as cells, casts, and crystals present in highly-pathological urine specimens. Thus, confirmation of such pathological results from automated devices by manual microscopic analyses may be useful.

\section{Acknowledgements}

The authors would like to thank Caroline Walker for English language editing.

\section{Potential conflict of interest}

None declared. 


\section{References}

1. Fuller $C E$, Threatte $G A$, Henry JB. Basic examination of the urine. In: Henry JB, Davey FR, Herman CJ, McPherson RA, Pincus MR, Threatte GA, Woods GL, eds. Clinical diagnosis and management by laboratory methods. 20th ed. Philadelphia: W.B. Saunders; 2001. p. 367-402.

2. Aspevall O, Hallander H, Gant V, Kouri T. European guidelines for urinalysis: a collaborative document produced by European clinical microbiologists and clinical chemists under ECLM in collaboration with ESCMID. Clin Microbiol Infect 2001;7:173-8. http://dx.doi.org/10.1046/j.1198743x.2001.00237.x.

3. Ono $Y$, Sugimoto $K$, Goto $M$, Kimura $M$, Kasai T, Shoji M, Yasujima M. Hematuria and acanthocyturia in patients with diabetes. Rinsho Byori 2005;53:993-8. (in Japanese.)

4. Memisogullari R, Yuksel H, Yildirim HA, Yavuz O. Performance characteristics of dipstick and microscopic urinalysis for diagnosis of urinary tract infection. Eur J Gen Med 2010;7:174-8.

5. Okada H, Sakai Y, Kawabata G, Fujisawa M, Arakawa S, Hamaguchi Y, Kamidono S. Automated urinalysis. Evaluation of the Sysmex UF-50. Am J Clin Pathol 2001;115:605-10. http://dx.doi.org/10.1309/RT7X-EMGF-G8AV-TGJ8.

6. National Committee for Clinical Laboratory Standards. Urinalysis and collection, transportation and preservation of urine specimens; Approved guideline. NCCLS document GP16-A. Villanova, PA, USA: NCCLS, 1995.

7. Kouri TT, Gant VA, Fogazzi GB, Hofmann W, Hallander HO, Guder WG. Towards European urinalysis guidelines. Introduction of a project under European Confederation of Laboratory Medicine. Clin Chim Acta 2000;297:305-11. http:// dx.doi.org/10.1016/S0009-8981(00)00256-4.

8. Regeniter A, Haenni V, Risch L, Kochli HP, Colombo JP, Frei R, Huber AR. Urine analysis performed by flow cytometry: reference range determination and comparison to morphological findings, dipstick chemistry and bacterial culture results. A multicenter study. Clin Nephrol 2001;55:384-92.

9. Ottiger C, Huber AR. Quantitative urine particle analysis: integrative approach for the optimal combination of automation with UF-100 and microscopic review with KOVA cell chamber. Clin Chem 2003;49:617-23. http://dx.doi. org/10.1373/49.4.617.

10. Roe CE, Carlson DA, Daigneault RW, Statland BE. Evaluation of the Yellow IRIS. An automated method for urinalysis. Am J Clin Pathol 1986;86:661-5. http://dx.doi.org/10.1093/ ajcp/86.5.661,

11. Simerville JA, Maxted WC, Pahira JJ. Urinalysis: a comprehensive review. Am Fam Physician 2005;71:1153-62.

12. Becker GJ, Garigali G, Fogazzi GB. Advances in urine microscopy. Am J Kidney Dis 2016;67:954-64. http://dx.doi. org/10.1053/j.ajkd.2015.11.011.

13. European Confederation of Laboratory Medicine. European urinalysis guidelines. Scand J Clin Lab Invest Suppl 2000;231:1-86.

14. Shayanfar N, Tobler U, von Eckardstein A, Bestmann L. Automated urinalysis: first experiences and a comparison between the Iris IQ200 urine microscopy system, the Sysmex UF100 flow cytometer and manual microscopic particle counting. Clin Chem Lab Med 2007;45:1251-6. http://dx.doi. org/10.1515/CCLM.2007.503.
15. Yuksel H, Kilic E, Ekinci A, Evliyaoglu O. Comparison of fully automated urine sediment analyzers H800-FUS100 and Labumat-Urised with manual microscopy. JCLA 2013;27: 3126. $h$ ttp://dx.doi.org/10.1002/jcla.21604.

16. Akin OK, Serdar MA, Cizmeci Z, Genc O, Aydin S. Comparison of LabUMat-with-UriSed and IQ200 fully automatic urine sediment analysers with manual urine analysis. Biotechnol Appl Biochem 2009;53:139-44. http://dx.doi.org/10.1042/ BA20080188.

17. Munson Ringsrud K, Jorgenson Linne J, eds. Urinalysis and body fluids: a colortext and atlas. 1st ed. St. Louis, MO: Mosby, 1995.

18. Roggeman S, Zaman Z. Safely reducing manual urine microscopy analyses by combining urine flow cytometer and strip results. Am J Clin Pathol 2001;116:872-8. http:// dx.doi.org/10.1309/GRT7-Q6WP-VGWE-OYUM.

19. Chien TI, Kao JT, Liu HL, Lin PC, Hong JS, Hsieh HP, Chien $M J$. Urine sediment examination: a comparison of automated urinalysis systems and manual microscopy. Clin Chim Acta 2007;384:28-34. http://dx.doi.org/10.1016/j. cca.2007.05.012.

20. Zaman Z, Fogazzi GB, Garigali G, Croci MD, Bayer G, Kránicz $T$. Urine sediment analysis: analytical and diagnostic performance of sediMAX - a new automated microscopy image-based urine sediment analyser. Clin Chim Acta 2010;411:147-54. http://dx.doi.org/10.1016/j.cca.2009.10.018.

21. Lamchiagdhase P, Preechaborisutkul K, Lomsomboon P, Srisuchart $P$, Tantiniti $P$, Khan-u-ra N, Preechaborisutkul B. Urine sediment examination: A comparison between the manual method and the IQ200 automated urine microscopy analyzer. Clin Chim Acta 2005;358:167-74. http://dx.doi. org/10.1016/j.cccn.2005.02.021.

22. Hannemann-Pohl K, Kampf SC. Automation of urine sediment examination: a comparison of the Sysmex UF-100 automated flow cytometer with routine manual diagnosis (microscopy, test strips, and bacterial culture). Clin Chem Lab Med 1999;37:753-64. http://dx.doi.org/10.1515/CCLM.1999.116.

23. Mayo S, Acevedo D, Quinones-Torrelo C, Canos I, Sancho M. Clinical laboratory automated urinalysis: comparison among automated microscopy, flow cytometry, two test strips analyzers, and manual microscopic examination of the urine sediments. J Clin Lab Anal 2008;22:262-70. http:// dx.doi.org/10.1002/jcla.20257.

24. Budak YU, Huysal K. Comparison of three automated systems for urine chemistry and sediment analysis in routine laboratory practice. Clin Lab 2011;57:47-52.

25. Dewulf G, Harrois D, Mazars E, Cattoen C, Canis F. Evaluation of the performances of the $i Q(\otimes) 200$ ELITE automated urine microscopy analyser and comparison with manual microscopy method. Pathol Biol (Paris) 2011;59:264-68. http://dx.doi.org/10.1016/j.patbio.2009.10.006. (in French)

26. Fenili $D$, Pirovano $B$. The automation of sediment urinalysis using a new urine flow cytometer (UF-100). Clin Chem Lab Med 1998;36:909-17. http://dx.doi.org/10.1515/ CCLM.1998.158.

27. Altekin E, Kadiçesme O, Akan P, Kume T, Vupa O, Ergor G, Abacioglu $H$. New generation IQ-200 automated urine microscopy analyzer compared with KOVA cell chamber. J Clin Lab Anal 2010;24:67-71. http://dx.doi.org/10.1002/ jcla.20319. 